\title{
¿Seguridad preventiva o acción punitiva? Las perspectivas del Plan Nacional de Vigilancia por Cuadrantes en Colombia ${ }^{1}$
}

https://doi.org/10.21830/9789585284869.01

Sara Patricia Quintero Cordero ${ }^{2}$ Ángela María Gaitán Navarro ${ }^{3}$

Escuela Militar de Cadetes "General José María Córdova”

\section{Introducción}

La historia, en su devenir y constante cambio en las sociedades, nos ha mostrado un panorama complejo en el que la violencia y la criminalidad resisten a la fuerza policiva y represiva del Estado. Surgen, en este sentido, nuevas modalidades de violencia en las distintas capas de la sociedad, y la respuesta del Estado ante el incremento de la inseguridad y de la percepción de riesgo por parte de los ciudadanos muestra su rostro más diverso, de forma que coexisten diferentes perspectivas de seguridad, en las que la acción punitiva y penalizadora del acto criminal convive con la acción preventiva del crimen.

1 Este capítulo hace parte de los resultados del proyecto de investigación "La construcción de la identidad nacional en Colombia a partir de la consolidación de la seguridad nacional" del Grupo de Investigación en Ciencias Militares, de la Escuela Militar de Cadetes "General José María Córdova" (ESMIC), registrado con el código COL0082556 de Minciencias. Los puntos de vista y los resultados de este artículo pertenecen a los autores y no reflejan necesariamente los de las instituciones participantes.

2 Internacionalista. Magíster en Relaciones Internacionales de la Universidad de Buenos Aires. Magíster en Seguridad y Defensa Nacionales de la Escuela Superior de Guerra "General Rafael Reyes Prieto". Docente e investigadora de la Facultad de Relaciones Internacionales de la Escuela Militar de Cadetes “General José María Córdova”. Orcid: https://orcid.org/0000-0002-9053-377X - Contacto: sara. quintero@esmic.edu.co

3 Profesional en Relaciones Internacionales y Estudios Políticos de la Universidad Militar Nueva Granada. Especialista en Educación Militar del Centro de Estudios Militares (CEMIL). Actualmente cursa la Maestría en Seguridad y Defensa en la Universidad Nebrija (España). Docente la Facultad de Relaciones Internacionales de la Escuela Militar de Cadetes “General José María Córdova”. Orcid: https:// orcid.org/0000-0002-0831-8246 - Contacto: angela.gaitan@esmic.edu.co 
Colombia es un claro ejemplo de la compleja situación de violencia y criminalidad. Como señala la Organización Panamericana de la Salud (Krug et al., 2002), América Latina se constituye en la actualidad como la región con mayor incremento en la taza de delitos en las ciudades, y Colombia es uno de los primeros de la lista en cuanto a homicidios, muertes violentas y otras modalidades de crimen. Además, los costos de la violencia en nuestro país no se limitan solo a sus víctimas; como señala Búvinic, esta "impide el desarrollo económico, [...] y genera gastos en el sistema policial y judicial” (1999, p. 9), sin contar, por supuesto, otros aspectos de orden social y cultural.

Una de las diversas respuestas del Estado colombiano frente a la ola de violencia y criminalidad en las ciudades es el Plan Nacional de Vigilancia Comunitaria por Cuadrantes (PNVCC). La evolución y transformación que ha tenido dicho programa mediante su consolidación en el 2010 permite visualizar una nueva estrategia del Estado en cuanto a políticas criminales. Cabe preguntarse, en efecto, si en este nuevo programa subsiste una perspectiva de seguridad preventiva o si, por el contrario, se trata de una perspectiva biunívoca de política criminal, definida por Delmas-Marty como de "coexistencia de la respuesta preventiva y la acción represiva y penalizadora” (1986, p. 19).

A lo largo de este capítulo se revisarán los principios y objetivos del PNVCC, el contexto en el cual se desarrolla y las acciones preventivas frente a las punitivas con que cuenta, de manera que se pueda comprender cuál es el accionar de la Policía Nacional frente al control de los delitos, las contravenciones y la garantía de la seguridad en los territorios.

\section{Nuevo modelo de gestión policial: PNVCC}

El PNVCC contiene un principio de cambios y transformaciones en las formas en que la Policía Nacional ejerce sus funciones y se relaciona la comunidad, por lo que se propone establecer relaciones de confianza con la población, de manera que puedan trabajar de forma articulada y velar conjuntamente por la seguridad de los territorios. Llevar a la práctica esta premisa hace posible que se promuevan acciones conjuntas con los habitantes de los territorios para generar mejores condiciones de convivencia, disminuir las contravenciones e instaurar acciones preventivas frente a los actos delictivos. 
El Plan se puso en marcha en julio del 2010, en ocho ciudades del país: Barranquilla, Bogotá, Bucaramanga, Cali, Cartagena, Cúcuta, Medellín y Pereira, como también en algunos municipios aledaños (Llorente et al., 2011). En los diferentes espacios territoriales se propuso implementar estrategias operativas que incidieran directamente en el incremento positivo de las percepciones de seguridad, para lo cual fue necesario emprender acciones orientadas a disminuir los índices de delitos y promover mecanismos que trajeran como resultado una buena convivencia entre las comunidades.

El PNVCC se propuso como una experiencia novedosa en el país, gracias a sus proyecciones, alcances, mecanismos de implementación y formas de gestión de la Policía Nacional. Por otra parte, su ejecución se estableció a través de una metodología progresiva, de manera que en las estaciones en las que el Plan entró primero en vigencia fueron aquellas que habían mantenido históricamente altos índices de contravenciones, conflictos y dificultades de convivencia, que eran las mismas en las que las percepciones de seguridad eran más bajas.

[El Plan] establece la necesidad del fortalecimiento de la estrategia en los municipios priorizados por la política, la focalización de la acción policial en las áreas de alta criminalidad en las zonas priorizadas y la modernización y unificación de los sistemas de comunicación y consulta de datos de la Policía. (Fundación Ideas para la Paz [FIP], 2012a, p. 2)

Con el fin de entender qué es el PNVCC y cuáles son sus alcances y proyecciones reales, a continuación se explica cuáles son sus principios, premisas y objetivos. A partir de esto, se podrá analizar mejor su estructura, la metodología de evaluación y los resultados que se han obtenido hasta el momento por medio de su aplicación.

\subsection{Componentes y principios del PNVCC}

La Policía Nacional implementó este Plan como una política de seguridad, a través de la cual se busca generar mecanismos de mejora y fortalecimiento de las relaciones de confianza entre la institución y los habitantes de los territorios. Por tanto, se pretende generar mecanismos para garantizar la seguridad y convivencia ciudadana, mediante el trabajo conjunto entre ambas partes. 
La implantación de la policía comunitaria en Bogotá es el resultado de una decisión interna de la propia Policía. [...] La iniciativa ha contado con el apoyo del mando policial y con la colaboración de la Cámara de Comercio de Bogotá [...]; el gobierno nacional a su vez, estableció como una de sus políticas centrales en materia de seguridad ciudadana la adopción del modelo comunitario por parte del conjunto de la institución. (Frühling, 2003, p. 17)

El funcionamiento del PNVCC depende de una estrategia y metodología de implementación, que fue diseñada con miras a cumplir el objetivo de optimizar el servicio y la efectividad de la Policía Nacional. A través de su implementación, se espera incrementar las percepciones de seguridad en cada una de las estaciones de las ciudades en las que el Plan se ha puesto en marcha. Con el fin de obtener mayor efectividad en el ejercicio de las funciones de la Policía Nacional, su formulación consta de cinco criterios base:

a) Alcanzar mayor interacción con el ciudadano

b) Contar con una respuesta oportuna a los requerimientos de la ciudadanía

c) Lograr una mejor integración con la comunidad

d) Generar corresponsabilidad frente a la seguridad ciudadana

e) Conocer detalladamente las causas y fenómenos que afectan el cuadrante (Policía Nacional, 2010a)

El PNVCC — como se establece de forma explícita en su nombrepropone una división del territorio por cuadrantes, con el fin de conocer de forma cercana las diferentes dinámicas que allí se establecen e instaurar relaciones más directas con la comunidad. Los cuadrantes son definidos como

un sector geográfico fijo, que a partir de sus características sociales, demográficas y geográficas, recibe distintos tipos de atención de servicio policial, entre los cuales se cuentan la prevención, la disuasión, control de delitos y contravenciones y la educación ciudadana en seguridad y convivencia. (Policía Nacional, 2010a, p. 24)

De esta forma, en los territorios geográficos delimitados se debe establecer una integralidad sobre las unidades del cuerpo policial que prestan el servicio de vigilancia en la zona y garantizan la seguridad, así como el control de contravenciones y la disminución de los delitos. Es por ello que los policías encargados de trabajar directamente con las comunidades deben estar capaci- 
tados para adaptarse a las transformaciones y cambios sociales que se presenten en el proceso, al tiempo que deben generar propuestas positivas en torno a sus funciones y formas de gestión.

Cada uno de los cuadrantes tiene asignadas patrullas con el personal necesario para cubrir tres turnos diferentes; sin embargo, teniendo en cuenta las características y dinámicas de cada territorio delimitado, se puede incrementar en número el personal de apoyo. Se espera, por lo tanto, que los policías que hacen parte de las patrullas tengan al menos una permanencia mínima de dos años. Es a partir de la continuidad en el tiempo de cada policía dentro del territorio que se logran generar los vínculos de confianza, credibilidad y responsabilidad que necesita el trabajo con la comunidad flotante y permanente del territorio.

Por otra parte, la información sectorizada por cuadrantes permite que se creen y adopten estrategias de prevención, atención especializada y ejecución efectiva de las políticas que conciernen al territorio. Además, permite evaluar la gestión y la forma de ejecución de las funciones de los policías que permanecen en los cuadrantes.

Con la implementación de dicha estrategia metodológica y de intervención de trabajo con las comunidades, se abre la posibilidad de conseguir información confiable sobre los problemas, contravenciones, conflictos y dinámicas recurrentes en el territorio, al tiempo que se plantean soluciones y se generan acciones de prevención y control para disminuir los actos delictivos. Es por ello que resulta trascendente involucrar en los procesos de implementación de planes de seguridad como los detallados a los habitantes del territorio y a la Policía, al tiempo que se incluyen las entidades e instituciones públicas (Llorente et al., 2011).

La relación con la comunidad se convierte en un eje transversal para el ejercicio de las funciones de la patrulla, ya que la aproximación integral hacia los dilemas del territorio debe hacerse con el acompañamiento de los directamente afectados. De igual forma, las soluciones que la Policía promueva y proponga a largo plazo para contrarrestar los conflictos que tienen una base estructural deben incluir a la población, teniendo en cuenta sus aportes e involucrándolos en los ejercicios de prevención. 
Aplicar el PNVCC como estrategia operacional de la Policía Nacional se establece con el objetivo de mejorar los resultados del manejo de los recursos institucionales y la capacidad de gestión para la resolución de conflictos y problemas de los territorios. Consecuentemente, se busca trabajar en las capacidades de respuesta integral por parte del Estado frente a las demandas, requerimientos y necesidades de la población en torno a la seguridad de los territorios. Se busca, por tanto, que los diferentes problemas que afectan la seguridad de los cuadrantes sean intervenidos por todas las entidades competentes a través de un ejercicio de cooperación y control por parte de las poblaciones.

El trabajo desarrollado para lograr tales alianzas constituye un avance notable hacia la efectividad y eficiencia de la prestación de servicios de la Policía Nacional para la comunidad. Al mismo tiempo, se trata de establecer proyectos en común con las entidades públicas y privadas, de la mano de la sociedad civil, con el fin de mejorar la calidad de vida de los y las colombianas.

En el trabajo coordinado con dichas instituciones (actores externos al cuadrante) y la comunidad se ponen en evidencia las capacidades de la Policía para entablar relaciones con los y las ciudadanas, proponer soluciones, mantener los lazos de confianza y cumplir los objetivos y metas señaladas en el PNVCC; en otras palabras, disminuir los actos violentos, el crimen, los delitos, los problemas y conflictos, lo cual configura el éxito y el nivel de impacto logrado.

Es por ello que entre los aportes principales del cambio administrativo del cuerpo de la Policía y la organización interna se encuentran el incremento de policías asignados a los espacios territoriales delimitados; las capacitaciones permanentes que apunten a mejorar la forma en que prestan el servicio y dan respuesta oportuna a la comunidad; y las formas de planeación, coordinación y supervisión de los servicios prestados a la ciudadanía.

Por otra parte, aquellos policías que implementen buenas prácticas y que presten un servicio eficiente a la comunidad son premiados por medio de una serie de estímulos como bonos, condecoraciones, distintivos, reconocimiento al mejor equipo de trabajo, al personaje del mes y permisos especiales. Este programa de estímulos pretende mantener un ambiente institucional positivo, que favorezca la calidad de vida laboral y el bienestar del cuerpo policial en 
general. Un valor agregado de esta estrategia para destacar los casos y experiencias exitosas es que se fomenta el sentido de pertenencia, para lo cual es fundamental mantener una cultura del reconocimiento e incentivar el fortalecimiento de las competencias vistas a nivel individual y colectivo.

Con el fin de fortalecer la aplicación y ejecución del PNVCC — que es un programa en desarrollo permanente-, el cuerpo policial debe encargarse de consolidar la información en unas memorias que incluyan los procesos con las comunidades. Esta información debe incluir la atención prestada por parte de las patrullas y las demás instituciones que se encuentren coordinadas. De esta forma, se obtiene valiosa información sobre las relaciones establecidas y las respuestas dadas a las inquietudes de la ciudadanía, gracias a lo cual se conocen claramente los precedentes para solucionar de manera efectiva futuros conflictos y problemas.

Para llevar a cabo la planeación participativa en los cuadrantes, es importante tener claro quiénes son los sujetos directamente involucrados en el proceso, de manera que se distribuyan de forma efectiva las acciones, según los grados de corresponsabilidad que se generen. Se espera, por lo tanto, que en los procesos mencionados participen de forma activa los habitantes del territorio, los policías que se encuentran encargados de velar por la seguridad y la buena convivencia en los cuadrantes, y las entidades u organizaciones que tienen algún nivel de relación con las dinámicas de los espacios geográficos delimitados.

Si bien el proceso de planificación e integración de la comunidad al PNVCC constituye uno solo de los componentes del Plan, es importante seguir una metodología clara, ya que del trabajo coordinado depende en buena medida la efectividad de las acciones emprendidas por la policía para mejorar las condiciones de seguridad y disminuir los índices delictivos. Así, se busca que el componente social sea parte fundamental del trabajo que realizan los policías en los cuadrantes, de manera que se establezcan procesos de perdurabilidad en los territorios de ejecución del PNVCC. De allí la importancia de que el cuerpo de la Policía se capacite en diferentes áreas, para poder asumir y responder cabalmente con los objetivos del Plan. 


\section{Metodología de evaluación y resultados}

La Fundación Ideas para la Paz (FIP) diseñó y planteó su programa de Seguridad Interior, Seguridad Ciudadana y Policial, a través del cual se propone analizar los alcances y las limitaciones detentadas en el PNVCC, reconociéndola como una política de seguridad ciudadana con una proyección prevista hacia la disminución de conflictos y contravenciones (Llorente et al., 2011).

Dentro de sus estrategias, y en concordancia con los principios del PNVCC, propende a la participación de los y las ciudadanas para realizar un trabajo conjunto, con el fin de promover el ejercicio de sus derechos y de convocar a la reflexión sobre la responsabilidad en materia de seguridad y el sentido de bienestar en los territorios. Por ello, las funciones de la FIP se concentran no solo en la evaluación, sino en ser parte estratégica del funcionamiento e impacto del PNVCC: las formas de evaluación y control, aparte de medir los resultados e impactos, se proponen como mecanismos para fortalecer las capacidades de los ciudadanos en la creación de redes y trabajo comunitario. Es allí en donde se concentra el valor agregado de la propuesta empleada.

El diseño de la metodología empleada se basa en una formulación experimental, que propone comparar resultados para estudiarlos en relación con la línea de base y el diagnóstico, los cuales fueron previamente elaborados en el propio territorio. De forma simultánea, esta metodología permite evaluar el impacto generado en las diferentes estaciones en las que PNVCC se ha puesto en marcha. La evaluación se realiza de forma sincrónica — simultáneamentey diacrónica —a través del tiempo- (FIP, 2011a). La metodología propuesta se encuentra dividida en dos componentes:

a. Monitoreo de la implementación del Plan frente a la gestión, disposición y actitud del cuerpo policial destinado a trabajar directamente en los territorios.

b. Medición y evaluación de impacto de los delitos, crímenes, problemas, rencillas y percepciones de seguridad de la ciudadanía.

Para la medición de la gestión de la Policía Nacional en la prestación del servicio y las relaciones establecidas con la comunidad, se formuló una meto- 
dología de seguimiento periódico, que busca indagar por las formas en que el policía asume sus funciones, entra en contacto con los habitantes del territorio y se establecen los vínculos de confianza necesarios para la efectividad y el éxito del PNVCC.

Por otra parte, el diseño y la implementación de la metodología propuesta por la FIP para la medición de los problemas, los conflictos de seguridad y la buena convivencia en los territorios proviene de la premisa de que si se establecen prácticas organizacionales de forma adecuada, entonces ello se verá reflejado en las acciones ejercidas por el cuerpo policial en el cumplimiento de sus funciones. Así, se espera que se logre un despliegue de competencias, actitudes requeridas y el uso correcto de las estrategias operativas y preventivas, a fin de generar un impacto en la disminución de delitos y mejorar las percepciones de la ciudadanía (Quintero Cordero, 2020).

Por lo tanto, se presupone que, mientras se promueve y se consigue aumentar la calidad de los factores implícitos en el PNVCC propuestos para su efectividad y eficacia, disminuirían los hechos criminales del sector. Así, se contemplan cuatro elementos clave que deben pasar por la evaluación de impacto:

a. Las prácticas de las organizaciones

b. El capital humano, entendido como el compromiso y las competencias adquiridas para poder estar en relación constante con la comunidad

c. La asignación efectiva de los policías en cada cuadrante

d. Los principios y procesos de las estrategias operativas, componente para el cual también se elaboró un diagnóstico previo y una línea de base

A partir de los primeros ejercicios de evaluación realizados en el 2011 en cuanto a la aplicación del PNVCC en los territorios, se ha encontrado que

En aquellas Estaciones de Policía en las cuales la estrategia ha sido bien implementada, es decir, donde el policía del cuadrante se ha acercado más a la comunidad, tiene un plan de trabajo, recibe retroalimentación y supervisión de sus superiores y trabaja en coordinación con otras entidades locales, las 
condiciones de seguridad han mejorado cuando se comparan con las condiciones de seguridad de aquellas estaciones donde el desarrollo del PNVCC va menos avanzado. (FIP, 2011b, p. 3)

Así mismo, la FIP pudo establecer que en aquellas estaciones en las que entró en vigencia el PNVCC se logró disminuir en un $11 \%$ el total de los delitos presentados, en comparación con los cuadrantes pertenecientes aquellas estaciones en las que no se había puesto en marcha. En cuanto a los principales problemas, conflictos y contravenciones de seguridad que se presentan en los territorios en los que se obtuvieron resultados positivos, se registraron los siguientes resultados:

a. Una reducción en los índices de las riñas en la vía pública

b. Una disminución de casos de registro de hurto de vehículos, residencias y establecimientos comerciales

c. Una reducción de la tasa de homicidios

d. Una disminución en el tráfico de armas y la ejecución de actos de extorción

Una de las principales estrategias del PNVCC es la forma de gestión por parte de la Policía al momento de hacer presencia en los territorios y dar respuesta efectiva e inmediata a sus necesidades. En efecto, a los cambios realizados en este sentido se les atribuye parte del éxito obtenido en las experiencias en los territorios. Es por ello que se puede hablar de una disminución importante de los delitos y un aumento en las percepciones de seguridad en comparación con aquellos cuadrantes en donde el Plan no ha entrado en vigencia y se mantienen los modelos tradicionales para garantizar la seguridad en los territorios (FIP, 2012a).

\section{Acciones preventivas frente a las acciones punitivas}

Como se ha visto hasta ahora, dentro del PNVCC priman las acciones preventivas sobre las represivas, por lo que el Plan apunta hacia los procesos de educación ciudadana, trabajo articulado con los habitantes del territorio, 
potencialización del capital humano y resolución de los conflictos por medio del reconocimiento de las diferentes dinámicas que se encuentran establecidas en cada uno de los cuadrantes. En otras palabras, se trata de buscar e identificar las causas y los factores que son reconocidos como generadores de los delitos, contravenciones, actos violentos y disturbios, de manera que sea posible realizar una serie de acciones directas de intervención para contrarrestarlos y eliminarlos.

[El PNVCC] se planteó como un servicio distinto al que tradicionalmente ha prestado la policía en el área de vigilancia (patrullaje) [...]. En contraposición, la modalidad comunitaria propuesta hacía hincapié en un enfoque preventivo y proactivo para enfrentar los problemas de seguridad ciudadana, basado en un trabajo de equipo entre la policía, la comunidad, y otras autoridades y organizaciones locales. (Llorente \& Rivas, 2004, p. 326)

Sin embargo, hay que tener en cuenta que el PNVCC es un proceso de larga duración y que, por sus características, hasta ahora está entrando en ejecución en todos los cuadrantes de las ciudades en las que se ha puesto en marcha como una estrategia nacional y local de seguridad. De este modo, resulta necesario e importante indagar por las propuestas y formas de ejecución de las acciones preventivas y punitivas que se encuentran en los principios y estrategias del Plan, así como por las formas de gestión de la labor realizada por los policías que hacen presencia en los territorios.

Las acciones preventivas se encuentran encaminadas a evitar los hechos y factores que inciden en la falta de seguridad ciudadana, por lo que es necesario generar mecanismos que permitan anticipar las causas que los generan. Es importante que los policías designados a cada territorio delimitado inicien procesos que faciliten identificar los riesgos para las condiciones de bienestar de las comunidades.

En la modificación del PNVCC, realizada en noviembre de 2010, se define la prevención como "toda acción orientada a identificar e intervenir de manera integral las causas y factores que generan inseguridad" (Policía Nacional, 2010a, p. 23). Para esto se propusieron siete estrategias puntuales:

1. Gestión interinstitucional o interagencial

2. Gestión comunitaria 
3. Creación y fortalecimiento de frentes de seguridad

4. Realización de encuentros comunitarios

5. Conformación y fortalecimiento de las redes de apoyo y comunicaciones

6. Fortalecimiento de la red de cooperantes

7. Elaboración y seguimiento de los informes de gestión ambiental, escombros y basuras, contaminación visual y alumbrado público

Para su correcto funcionamiento, es importante la identificación de las causas originadoras pero también de las causas facilitadoras. Las primeras son identificadas como aquellas que son consecuencia directa de las dificultades y conflictos sociales generados por la insatisfacción de las necesidades básicas las comunidades. Algunos de estos problemas son el desempleo, la falta de educación y de oportunidades y la pobreza. Por su parte, las causas facilitadoras son

Todas aquellas acciones u omisiones del individuo con relación a su rol social, que permiten preparar y ejecutar con menor dificultad los crímenes, las contravenciones y los conflictos ciudadanos por parte del victimario, haciéndose más fácil su comisión o repetición, tales como la indiferencia social, falta de cooperación, el miedo común, etc. (Policía Nacional, 2010a, p. 25)

Además, se trata de indagar por los factores socioculturales y estructurales directa o indirectamente involucrados con las dinámicas de los territorios y sus problemas, conflictos y contravenciones. De esta manera, se pueden generar acciones preventivas a largo tiempo, que incidan directamente en las problemáticas señaladas y en las causas previamente identificadas.

Los factores estructurales se encuentran relacionados con aquellas insuficiencias y debilidades del entorno físico del sector que influyen en las causas de los hechos delictivos o criminales. En otras palabras, los factores estructurales son aquellos que le brindan al escenario unas condiciones adecuadas y acordes para que se presenten hechos que alteren la seguridad y afecten la buena convivencia de los cuadrantes, por ejemplo, los callejones mal iluminados, los parques abandonados, los lotes descuidados, el mal alumbrado público, las basuras y el mal estado de la malla vial, entre otros.

Los factores socioculturales, por su parte, tienen que ver con aquellas acciones u omisiones colectivas que se encuentran fuertemente arraigadas a las 
costumbres y que, por lo general, están ligadas a aquellas prácticas definidas como de mala costumbre, es decir, van en contra de las leyes y normas morales o éticas socialmente aceptadas, mientras que son practicadas directa o indirectamente por la mayoría de las personas que conforman las comunidades. Entre estas, se encuentra la cultura de la ilegalidad, que hace referencia principalmente a la compra y venta de productos cuya procedencia no es legítima. Ante hechos de este tipo, la respuesta de la ciudadanía suele ser de indiferencia social, apatía o descuido (Quintero Cordero \& González, 2017).

\section{Plan Nacional de Inteligencia Comunitaria por Cuadrantes}

Con el objetivo de cumplir y abarcar los aspectos mencionados anteriormente, el PNVCC se apoya en el Plan Nacional de Inteligencia Comunitaria por Cuadrantes (PNICC). Puesto que las estrategias de inteligencia constituyen uno de los cuatro procesos misionales de la Policía, resulta imperioso que se incluya en el PNVCC de manera positiva y acertada, para que pueda contribuir a las acciones emprendidas con el fin de garantizar la seguridad de los territorios y la buena convivencia de los ciudadanos (Policía Nacional, 2010a).

Con el objetivo de ajustarse a los requerimientos y principios del Plan, las estrategias de inteligencia se han propuesto adecuar su plataforma operacional con el fin de generar mecanismos de inteligencia que sean primordialmente preventivos y tengan la capacidad de anticiparse a la multiplicidad de expresiones de violencia y criminalidad usuales en los territorios.

El PNICC, además de generar acciones para prevenir y eliminar los aspectos asociados a la criminalidad de los territorios delimitados, enfatiza sus acciones y análisis en las diferentes dimensiones que en algún momento puedan generar condiciones que afecten la seguridad y sana convivencia de las poblaciones. Por lo tanto, su trabajo se encuentra dirigido a identificar y trabajar positivamente en las percepciones de seguridad, las dinámicas sociales, la protección del medioambiente, los fenómenos criminales y los factores económicos (Quintero Cordero \& Molina Orjuela, 2013). 
Esta estrategia de reorientación de la inteligencia policial pretende generar conocimientos sobre los fenómenos de carácter delincuencial o potencializadores de los conflictos sociales, cuyas consecuencias se pueden observar en lo nacional, lo regional y lo local —es decir, en los cuadrantes, por lo cual resultan apropiados los principios propuestos en el PNCC de atención oportuna, cercana y especializada en las dinámicas de los territorios delimitados-.

Así, para la implementación del PNICC, se propone el diseño y la formulación de un modelo de inteligencia policial que, a su vez, funcione por cuadrantes, teniendo en cuenta las dinámicas y el diagnóstico previamente elaborado con las comunidades y los habitantes de los territorios. De esta manera, el PNICC estará orientado a la recolección de la información de zonas específicas y delimitadas, para generar acciones de prevención de aquellas situaciones causantes de inseguridad, conflictos y contravenciones. En otros términos, se plantea que,

mediante la identificación temprana de factores generadores de riesgo, [se establezca] una inteligencia accionable que, a través de una alta capacidad de recolección de información, producción y difusión de inteligencia, se anticipa a la comisión de conductas delictuales y restablece los niveles de seguridad. (Policía Nacional, 2010a, p. 39)

De esta manera, por medio de la ejecución del PNICC, se espera obtener conocimiento importante y necesario sobre las dinámicas de los territorios, cuyo contenido principalmente sea de carácter estratégico y accionable, que permita la comprensión de los problemas y conflictos estructurales, junto con su evolución y las alternativas integrales propuestas como mecanismos de control y eliminación de las causas generadoras de problemas identificadas dentro del proceso.

El PNICC se establece como un componente importante dentro del Plan, ya que contribuye de manera efectiva a la convivencia ciudadana y la seguridad de los territorios. De hecho, dentro de sus principios se establece brindar apoyo oportuno a las demás instituciones y cuerpos de la policía, con el fin de contribuir a la eliminación de la impunidad, los actos delictivos y criminales. La estrategia de inteligencia contribuye, además, a la seguridad preventiva, ya que sus mecanismos de investigación permiten enfocar los 
esfuerzos en las causas de los problemas (factores y dimensiones) que necesitan una intervención directa por parte de la Policía en un trabajo articulado con las comunidades (Quintero Cordero \& Molina Orjuela, 2013).

\subsection{Tabla de Acciones Mínimas Requeridas}

De acuerdo con la metodología y el proceso de evaluación implementados por la FIP, en el PNVCC se encuentra propuesta la utilización de la Tabla de Acciones Mínimas Requeridas (Tamir), como instrumento que permite organizar y guiar el trabajo de las patrullas en los cuadrantes. Esta herramienta es considerada como un instrumento básico que apoya el proceso y los mecanismos de planeación, ejecución y evaluación del servicio prestado por parte de la Policía Nacional en los cuadrantes. En este instrumento se consignan todas las actividades realizadas en relación directa con las metas de desempeño, la ejecución previamente acordada y las estrategias de prevención implementadas.

Es así que el registro se divide en tres unidades (Policía Nacional, 2010a) que se explican a continuación. La primera unidad se destina a las actividades de prevención y disuasión: las campañas educativas, la gestión comunitaria, la capacitación a la policía cívica de menores, las reuniones realizadas con los líderes de frentes de seguridad, los informes de inteligencia, los reconocimientos de las fuentes comunitarias de información, los actos de control, las acciones de orientación a la ciudadanía, la intervención en comunidades específicas, el contacto con cooperantes, la regulación del tránsito, los informes especiales y la gestión ambiental (Quintero Cordero, 2020).

La segunda unidad está destinada a registrar las acciones de corresponsabilidad, que incluyen las campañas como Desarma tu Corazón, Entornos Viables y Seguros, Concierto por la Vida, Por mi Bella Ciudad, Por una Ciudad Segura y Por mi Buen Barrio.

La tercera unidad concentra las acciones específicas de control, que incluyen las actividades operativas, la incautación de armas de fuego y estupefacientes, la recuperación de automotores y mercancía, y las capturas en flagrancia y por orden judicial. 


\subsection{Programas de prevención inscritos en el PNVCC}

Los programas que se encuentran en el PNVCC destinados al área de prevención y educación ciudadana son los siguientes: campañas de gestión comunitaria, campañas educativas, encuentros comunitarios, escuelas de seguridad, frentes de seguridad, gestión interinstitucional, policía cívica de mayores, policía cívica juvenil, red de apoyo y comunicaciones, red de apoyo y solidaridad y red de cooperantes (Policía Nacional, 2010a).

Todos los programas mencionados enfatizan sus acciones e intereses en sectores y problemáticas puntuales; sin embargo, tienen en común hacer partícipes a las comunidades en los procesos, de manera que se tiende a los mecanismos de participación y articulación con los habitantes del territorio (Quintero Cordero \& Molina Orjuela, 2013).

Para llevar a cabo y ejecutar los mecanismos de participación ciudadana en los cuadrantes, es importante tener claro quiénes son los sujetos directamente involucrados en el proceso, de manera que se distribuyan de forma efectiva las acciones según los grados de corresponsabilidad que se generen. Así, se espera que, en los procesos que se ponen en marcha con el fin de disminuir los actos violentos y las contravenciones, al tiempo que aumentan los índices de percepción de seguridad, participen de forma activa los habitantes del territorio, los policías encargados de velar por la seguridad y la buena convivencia en los cuadrantes, y las entidades u organizaciones que tengan algún nivel de relación con las dinámicas de los espacios geográficos delimitados.

Si bien el proceso de planificación e integración de la comunidad al PNVCC constituye uno solo de los componentes del Plan, es importante seguir una metodología clara, ya que del trabajo coordinado depende en buena medida la efectividad de las acciones emprendidas por la Policía para mejorar las condiciones de seguridad y disminuir los índices delictivos. Se busca, de esta manera, que el componente social sea parte fundamental del trabajo que realizan los Policías en los cuadrantes, con el fin de que haga parte de las acciones preventivas. Es así como se establecen procesos de perdurabilidad en los territorios, que requieren un tiempo de ejecución constante del PNVCC. De allí la importancia de que el cuerpo de la Policía se capacite en diferentes áreas para poder asumir y responder cabalmente a los objetivos del Plan. 
El desarrollo metodológico establece las pautas para orientar el servicio de policía en todos los niveles de despliegue. Parte de la definición de los verbos rectores marcan la ruta que orienta la implementación de las tres fases, así: fase 1: comprensión contextual del territorio (comprender, identificar, caracterizar, priorizar); fase 2: intervención y coordinación (intervenir); fase 3: evaluación y seguimiento (evaluar), desplegada en los niveles estratégico, táctico y operacional. (Policía Nacional, 2014, p. 26)

El proceso de planeación, desarrollo y ejecución de los diferentes programas que buscan involucrar y articular a las comunidades se convierte en un eje central para las acciones preventivas con el fin de disminuir las acciones punibles que se desarrollan en el marco de la respuesta y la solución inmediata, como se explica a continuación.

Como se puede observar, las diferentes estrategias promovidas en la implementación de un enfoque preventivo por parte de la Policía Nacional responden a un esquema asociado a la seguridad democrática, a partir de la cual se pone de relieve la importancia de garantizar la tranquilidad y el bienestar de los individuos en el territorio. En este sentido, se observa un cambio frente a los mecanismos empleados al momento de enfrentar y contrarrestar los actos delictivos, criminales y contravenciones que ponen en riesgo el bienestar de las comunidades. La Policía Nacional, en consecuencia, ha puesto en marcha diferentes estrategias para estar en concordancia con los principios (incluidos en el PNVCC) de esta política de seguridad preventiva.

El modelo de seguridad preventiva, como su nombre lo indica, tiene como principal objetivo la actuación antes de que ocurran los hechos, de manera que propone una estrategia que prioriza la inteligencia. Esto lo hace a través de la indagación de las causas originarias y el diagnóstico de cada cuadrante. A lo anterior se le suma el ejercicio diario de registro de actividades, gracias al cual es posible hacer un seguimiento de las acciones emprendidas por los policías que hacen presencia en los territorios y de los resultados obtenidos, así mismo, se ubican las actividades diarias que se realizan con las comunidades.

En consecuencia, es importante que los mecanismos empleados por la institución —como los mencionados hasta el momento-, el PNICC y la Tamir fortalezcan y optimicen la implementación de cada uno de los programas diseñados para garantizar la seguridad desde una perspectiva preventiva antes que reactiva. 


\subsection{Acciones punibles}

Las acciones punibles están relacionadas con los hechos susceptibles de ser sancionados por la ley como un delito, es decir, aquellos cuyo accionar provoca un daño a una tercera persona. Las leyes y normas que se dictan para castigar tales actos tienen como objetivo principal proteger la seguridad pública, mantener la sana convivencia entre los ciudadanos y garantizar la seguridad en el territorio nacional (Velázquez, 2000).

Es así que dentro del PNVCC — y dentro de cualquier estrategia encaminada a garantizar la seguridad de los territorios y de la ciudadanía-, las acciones o hechos punibles se encuentran ligados a los castigos aplicados a quienes cometan actos en contra de la ley. En el PNVCC, este tipo de acciones se encuentra definido como "acto o conducta antijurídico, típico y culpable que merece la imposición de una pena o castigo" (Policía Nacional, 2010b, p. 124). De esta manera, se infiere que, si bien hay una predisposición a generar procesos encaminados a la prevención como forma de control de los delitos, subsisten mecanismos que se encuentran asociados con la represión por medio del castigo penal y judicial.

Los modelos tradicionales estuvieron encaminados a mantener mecanismos de reacción y control de delitos de manera reactiva; esto significa que las instituciones encargadas de garantizar la seguridad en los territorios reaccionan a los sucesos luego de que estos han tenido lugar, es decir, sus acciones se dirigen a encontrar y culpabilizar a los actores de los hechos. Por el contrario,

las nuevas tendencias intentan transformar la conducta policial en proactiva, en el sentido que los policías pueden llevar a cabo tareas sumamente enriquecedoras para la Comunidad, como lo sería recabar datos acerca de las problemáticas existentes en el barrio. El policía como profesional de la seguridad, es un estudioso de las circunstancias sociales que generan la emergencia del desorden, el delito y el temor al delito. (Risco, 2009, p. 6)

Esto quiere decir que, en los nuevos modelos de seguridad — dentro de los que se encuentra inscrito el PNVCC—, los policías que hacen presencia en los territorios se encargan de conocer las dinámicas, de mapear los conflictos y contravenciones más frecuentes y de indagar por las causas originarias de los hechos. Así, pues, sus acciones se realizan antes de que los hechos punibles y 
delictivos tengan lugar, por lo tanto, se hace referencia a las acciones preventivas explicadas anteriormente en las que participa la comunidad, para la solución de los conflictos y problemas de los cuadrantes.

Por otra parte, en los modelos tradicionales (en los que prima el control de los delitos mediante la aplicación de la ley frente a los actos delictivos), los policías mantienen una forma de gestión vertical, es decir, las decisiones se toman jerárquicamente, por lo que los habitantes de los territorios no tienen autonomía para decidir sobre los hechos a los que se enfrentan, sino que están sujetos a decisiones que han sido tomadas con anterioridad. Tales modelos implican un orden de actuación y operación por parte de la Policía en un marco descontextualizado de las dinámicas de cada territorio.

\section{Plan Nacional de Investigación Criminal por Cuadrantes}

De la misma manera que el PNVCC contiene el Plan Nacional de Inteligencia Comunitaria por Cuadrantes (PNICC) para fortalecer las formas de gestión de la Policía en su accionar preventivo, también instaura el Plan Nacional de Investigación Criminal por Cuadrantes (PNIPC), como mecanismo de acción frente a los hechos punibles. Mantener ambas perspectivas le da una mayor coherencia al Plan, de manera que puede desarrollar adecuadamente los procesos emprendidos para promover la prevención antes que la actuación frente a los hechos delictivos.

[El PNIPCC] tiene como objetivo articular el proceso misional de Investigación Criminal al PNVCC y el PNIPC, con el fin de contribuir de manera efectiva a la seguridad y convivencia ciudadana, orientada a brindar apoyo oportuno a la administración de justicia en la lucha contra la impunidad. (Policía Nacional, 2010a, p. 61)

Por lo tanto, el PNIPC y PNICC se convierten en una estrategia integral que hace parte del PNVCC con el fin de garantizar la seguridad de los territorios, al tiempo que apoya los procesos de larga duración emprendidos a favor de las acciones de prevención. Con la articulación de PNICC y PNIPC, el PNVCC se concentra en tres ámbitos o dimensiones de actuación específicas. 
La primera tiene que ver con la cobertura, es decir, los procesos de investigación criminal emprendidos por la Policía Nacional generalmente se encuentran estructurados a partir de un nivel central, con el fin de operar en todo el territorio nacional a través de unas unidades de actuación desconcentradas, como las Seccionales de Investigación Criminal. Esto significa que en el proceder del cuerpo policial se ha detectado la necesidad de optimizar y fortalecer la presencia de la institución encargada de velar por la seguridad y el bienestar de la ciudadanía, especialmente, en aquellos territorios en donde los actos criminales y delitos ascienden cada vez más y generan altas percepciones de inseguridad entre las comunidades.

La segunda dimensión es la de oportunidad, que tiene que ver con el desarrollo de las capacidades de la institución para hacer frente a los problemas y delitos por medio de la aplicación de Laboratorios Regionales de Policía Científica y Criminalística, cuyo proyecto es desarrollado por la Dirección de Investigación Criminal de la Interpol en el marco de la Ley 906 de 2004.

Acá se busca desconcentrar y descentralizar la prestación de los servicios de la Policía para darle celeridad y prontitud a los requerimientos y necesidades de las comunidades, por medio del uso de tecnologías avanzadas, en las que se concentran las experiencias y la información total de los hechos, de forma que se logre establecer las conexiones que existen entre los actos delictivos y criminales y los autores o participantes de los hechos.

La tercera dimensión es la tecnológica, en la cual se hace uso de las innovaciones en investigación criminal, para dar respuesta inmediata cuando suceden los hechos, al tiempo que se espera lograr una reducción efectiva de los actos delictivos y criminales.

Por otra parte, el PNICC se encuentra encargado principalmente de cuatro acciones específicas, establecidas en el PNVCC (Policía Nacional, 2010a, p. 35):

a. Capturas en flagrancia: Se trata de dar respuesta inmediata durante el tiempo mismo en que se están realizando las acciones delictivas en los territorios geográficos delimitados. Se da captura inmediata y se recogen las pruebas necesarias para la posterior judicialización y aplicación de la ley. 
b. Remisión de detenidos: Una de las acciones a tomar por parte de los policías que integran los cuadrantes es el traslado de personas privadas de la libertad de un lugar a otro, realizando una labor de protección de la persona detenida y de la comunidad o los habitantes del territorio.

c. Incautación de mercancía: El equipo de la Policía que hace presencia en los territorios, cuyas funciones se encuentran relacionadas con las formas de gestión de reacción, están encargados de realizar las diversas incautaciones de elementos que circulan de forma ilegal, es decir, aquellos cuya procedencia se desconoce o no se encuentra debidamente legitimada, por ejemplo, mercancías y combustibles portados sin permiso legal.

d. Conducciones preventivas: Se refiere al acompañamiento realizado por los policías de cada cuadrante a una persona en un alto estado de ebriedad, con el objetivo de evitar posibles riñas o malas conductas. Se trata acá de la protección de la persona implicada y de los habitantes del territorio, con el fin de garantizar su seguridad y las condiciones óptimas de convivencia.

El registro de las acciones por el PNICC es, en parte, lo que permite definir si el PNVCC ha cumplido con los objetivos en cada cuadrante, ya que se realiza una medición del registro de los delitos y actos criminales junto a las acciones de intervención directa de la Policía en el momento en el que se producen los hechos.

\section{Hojas de Servicio}

En la metodología del proceso de evaluación del PNVCC implementado por la FIP, se encuentra propuesto el uso de las Hojas de Servicio, documento en el cual

el coordinador del cuadrante plasma las consignas, puntos críticos, misiones, tareas a desarrollar y un análisis estadístico de la parte delictiva y operativa, indicando días y horarios de mayor afectación de cada cuadrante, esto como 
elemento orientador para la prestación del servicio de las patrullas. (Policía Nacional, 2010a, p. 31)

Los instrumentos de recolección de información (Tamir y las Hojas de Servicio) permiten una evaluación integral del accionar de la Policía, es decir, valorar las acciones en el campo preventivo y de reacción. Además, con la información recogida y registrada en cada herramienta es posible evaluar puntualmente cada tipo de accionar y convertirlo en un insumo para mejorar procedimientos y, a su vez, la política criminal del Estado.

En este sentido, las Hojas de Servicio contienen información que se establece de manera puntual dentro de las acciones de reacción, es decir, aquellas formas de actuación de la Policía que se dan en el mismo momento en que se producen los hechos delictivos con carácter punible. Entre los puntos más relevantes a ser consignados en el instrumento se encuentran los siguientes:

a. Estadísticas de la semana anterior frente a las acciones operativas y delictivas del cuadrante

b. Un análisis estadístico sobre los hechos delincuenciales y de contravención, con la clasificación de los días en que se registra mayor afectación

c. Un análisis estadístico sobre los hechos delincuenciales y de contravención, con la clasificación de las horas en las que se registra una mayor afectación

d. Según los análisis realizados, se ubican los puntos críticos de cada cuadrante, de manera que la información contribuya a las acciones de prevención que se formulen para actuar en cada espacio específico

\section{Programas y áreas de intervención para enfrentar las acciones punibles contenidas en el PNVCC}

Los programas y áreas de intervención que se encuentran en el PNVCC destinados a enfrentar las acciones y hechos delictivos, cuyo carácter se define como punible, son: el control de disturbios, los eventos electorales, las infrac- 
ciones cometidas por personas con fuero, el uso indebido del espacio público, las manifestaciones, los desalojos por orden de autoridad competente y la incautación de armas de fuego, municiones y diferentes elementos cuya apropiación por parte de la ciudadanía sea de carácter ilegal.

Dentro de las estrategias generales del PNVCC, se encuentra la necesidad de promover la respuesta inmediata para enfrentar las acciones delictivas que se presenten en los territorios; de manera que en la formulación y el diseño del Plan se observa la necesidad de promover estrategias preventivas y todas aquellas que contrarresten los comportamientos punibles. Así, los diferentes programas y las áreas de intervención que hacen parte de los mecanismos para enfrentar las acciones punibles se mantienen vigentes y funcionan de forma paralela con las estrategias y propuestas encaminadas a orientar las acciones de prevención.

\section{Nuevo modelo de gestión policial: ¿̨prelación de acciones preventivas o punitivas?}

El PNVCC se puso en marcha con el fin de mejorar las condiciones de seguridad del territorio, mediante la modificación de las formas de gestión con que los Policías hacen presencia en la ciudad y entran en relación con las comunidades; de allí que la propuesta inicie con la sectorización del espacio por cuadrantes, al tiempo que establezca una forma de atención personalizada, cercana y articulada con la población para dar respuesta a sus necesidades y requerimientos.

En este sentido, en su formulación se cuenta con una serie de estrategias que propenden a la articulación de la labor de la Policía con la comunidad, es decir, se quiere generar mecanismos para que la seguridad sea entendida como un bien común y público, que hay que velar y proteger. De la misma manera, el Plan propone la asociación con actores clave para la solución de los problemas y contravenciones que se presentan en los cuadrantes.

A partir de estas diferentes características que forman parte integral del PNVCC, se puede observar que la finalidad es garantizar la seguridad de los territorios por medio de la implementación de un nuevo modelo de seguridad. Como su nombre lo indica, la gestión de la institución encargada de velar por 
el bienestar y la estabilidad de los territorios se propone con una perspectiva de policía comunitaria, la cual se entiende como

una filosofía y una estrategia organizacional que promueve una nueva asociación entre la comunidad y su policía. Está basada en la premisa de que ambos deben trabajar en conjunto para identificar, priorizar y resolver los problemas actuales [...] con el objetivo de mejorar la calidad de vida en la zona. (Montbrun, 2002, p. 5)

Una de las principales implicaciones de la ejecución del nuevo modelo es la ampliación de las tareas de la Policía, ya que, aparte de cumplir con las funciones básicas establecidas en los modelos tradicionales de seguridad (patrullaje constante preventivo, operativos de control, respuesta inmediata a los requerimientos, entre otras), deben asumir nuevas tareas en relación con las formas de organización y articulación con las comunidades.

Entre estas actividades se encuentran la prevención proactiva de los conflictos, crímenes y delitos; la generación de mecanismos de voluntariado; el trabajo organizado con fundaciones e instituciones que tengan algún tipo de inherencia en los territorios; la participación en los procesos de mediación y facilitación; y el contacto directo y permanente con otros organismos del gobierno en favor de los habitantes del territorio, entre otras.

Sin embargo, como se observa a lo largo del documento, desde el mismo planteamiento, diseño y formulación del PNVCC, se establece explícitamente la ejecución de acciones preventivas — relacionadas con el nuevo modelo de policía comunitaria- y de acciones punitivas, las cuales se encuentran dentro de los modelos tradicionales de policía reactiva. A partir de la promoción y ejecución de ambas perspectivas, se propone el objetivo de garantizar la seguridad en los territorios, mejorar las percepciones de seguridad y promover las condiciones necesarias para mantener una buena convivencia entre la ciudadanía.

\section{Diferencias entre las propuestas de acciones preventivas y acciones punitivas}

Tanto los modelos tradicionales de seguridad como las nuevas propuestas en las que se involucra a las comunidades para generar trabajos articulados 
mantienen un mismo objetivo: garantizar la seguridad del territorio y brindar bienestar a la ciudadanía. Teniendo esto claro, es posible afirmar que las diferencias se encuentran suscritas en las formas de gestión con las cuales cada modelo se propone enfrentar y controlar los actos delictivos y criminales que afectan la seguridad de las comunidades.

En este sentido, las principales características de los modelos de seguridad tradicional frente a las nuevas formas de gestión de la policía comunitaria — de acuerdo con los principios formulados en el PNVCC — cubren las siguientes nueve dimensiones básicas:

1. Intervención reactiva frente a intervención proactiva

2. Procesos centralizados frente a procesos descentralizados

3. Principio de respuesta unificada, proveniente de las jerarquizaciones de la Policía, frente a respuestas múltiples, gracias a la capacidad de autonomía de los policías que hacen presencia en cada cuadrante

4. Resultados evaluados por medio de índices numéricos frente a una evaluación realizada a partir de conceptos y procesos

5. Trabajo por la ciudadanía frente a un trabajo con la ciudadanía

6. Estructura jerárquica frente a una estructura heterárquica, en donde se propende hacia la construcción de redes

7. La dirección de su gestión pone énfasis en tareas, frente a un direccionamiento relacionado con los resultados

8. Provee respuestas a partir de las normas, frente a la generación de respuestas por medio de procesos articulados con las comunidades

9. Principio exclusivo, según el cual el policía se mantiene al margen de los procesos de la ciudadanía, frente a un principio inclusivo, en el que se da lugar a una integración del cuerpo de la Policía con los habitantes de la comunidad

Como es evidente, las acciones propuestas en el PNVCC se diferencian de los modelos tradicionales por poner énfasis en la prevención y el trabajo articulado con las comunidades, para garantizar su seguridad y mantener condiciones estables para la buena convivencia; es por ello que se desataca el trabajo articulado con las comunidades y la solución de los problemas por medio de la incidencia en su causas, no en sus consecuencias. 
En este sentido, se resalta la intervención proactiva por parte de los policías, su capacidad de autonomía frente a las limitaciones de los mandatos jerárquicos, los procesos descentralizados, la evaluación realizada por conceptos y procesos, la construcción de redes y la generación de respuestas por medio de procesos articulados con las comunidades. Así mismo, se trascienden las nociones y principios de los modelos tradicionales, como la intervención reactiva, los procesos centralizados, la prevalencia de estrategias militares, la respuesta única y unificada (es decir, descontextualizada), el trabajo por la ciudadanía de forma exclusiva y los obstáculos de la estructura jerárquica.

Teniendo en cuenta que los modelos de seguridad deben ser acordes y ajustarse a los contextos y a las nuevas circunstancias provenientes de las transformaciones sociales y políticas, resulta altamente beneficioso que tanto el Estado como la institución policial adopten nuevos mecanismos, principios y propuestas que tengan la capacidad y flexibilidad de ajustarse a los requerimientos y las necesidades de cada contexto y territorio (Ripoll de Castro \& Quintero Cordero, 2018).

El modelo de seguridad preventiva del PNVCC va de la mano con las nuevas tendencias latinoamericanas de seguridad, en las que prevalecen las comunidades, se proponen mecanismos que generen procesos fortalecidos para garantizar la seguridad en los territorios y las respuestas a los conflictos se dan por medio de procesos participativos con la sociedad. Se trata, entonces, de poner en marcha mecanismos de prevención, en los que se superen las estrategias reactivas y punitivas para dar respuesta a un hecho en el momento que ocurre. De esta forma, si se sigue la línea punitiva (en lugar de arriesgarse por la prevención, la restricción a la libertad, el castigo o el reproche establecido en el código penal), no será posible generar procesos de prevención, que son los que verdaderamente evitan la ocurrencia de acciones delictivas.

De esta forma, se pretende traspasar la noción de que los delitos o actos criminales no se realizan por temor a la pena o castigo que reciben los culpables; por el contrario, se abre la posibilidad de que, a partir de los mecanismos y estrategias de prevención, los hechos no se cometan por dos razones principales: primero, porque no se dan las condiciones para que ello ocurra y, segundo, porque se tiene la conciencia de que es un acto que está mal en sí mismo, más allá del castigo. 


\section{De las acciones punitivas a las acciones de prevención}

A partir del análisis realizado, se puede establecer que el cambio en las formas de gestión para garantizar la seguridad en los territorios, a partir de la implementación del nuevo modelo propuesto por la Policía Nacional, depende de un proceso de transición en el que se encuentren implicados de igual manera la institución y la ciudadanía. Es por ello que la ejecución del Plan depende de una estrategia de implementación progresiva en las ocho ciudades en las que se ha puesto en marcha: los cuadrantes en donde se ha puesto en funcionamiento de manera prioritaria son aquellos que responden a dinámicas altamente conflictivas, lo que da relevancia a los resultados obtenidos (FIP, 2012b).

Mantener una forma de ejecución de los modelos tradicionales, al tiempo que se instauran las nuevas formas de gestión de la policía comunitaria, permite generar un apoyo efectivo mientras se produce el proceso de transición de un modelo al otro. Esto hace que se fortalezcan los procesos de articulación comunitaria y se optimicen los resultados en favor de la disminución de los delitos, de problemas y contravenciones, al tiempo que aumentan los índices de las percepciones de seguridad.

Entre las principales formas de gestión que es importante empezar a modificar para garantizar una implementación de acciones preventivas de manera prioritaria sobre las punitivas, se encuentra la manera en que se efectúa el control de los delitos: gracias a la implementación del modelo de la policía comunitaria, se propone una indagación previa que contrarreste las causas originarias. De forma muy diferente con los modelos reactivos, que proponen una actuación principalmente sobre los hechos en marcha, con el objetivo de capturar a los delincuentes en flagrancia, sin importar la eliminación de las causas generadoras de los hechos.

Desde esta perspectiva, se puede inferir la necesidad de trabajar sobre diferentes requerimientos de las comunidades que trascienden la misma noción de seguridad. Por esta razón se habla de la importancia de generar procesos articulados en los que se promueva el bienestar de los habitantes del 
territorio junto al mantenimiento de las condiciones estables para garantizar una sana convivencia entre la ciudadanía. En este sentido, el nuevo modelo de policía comunitaria se establece bajo principios proactivos. Esto implica que el Policía que hace presencia en los territorios y entra en contacto directo con las comunidades se convierta en un profesional de la seguridad, esto quiere decir que, entre sus funciones, debe primar el estudio, análisis y conocimiento de las circunstancias, las condiciones y las dinámicas del cuadrante al que es asignado.

A partir de allí, es posible que empiece a involucrarse efectivamente con las comunidades para generar procesos articulados y así contrarrestar las contravenciones. Para ello, deben primar las formas preventivas de los problemas, conflictos, contravenciones y actos delictivos que tienen alta frecuencia en el territorio, con el fin de disminuir los sucesos que necesitan una intervención directa y mejorar la seguridad en los territorios.

El policía conoce, mapea, estudia y diagnostica una situación social (en un barrio, una comunidad, un grupo étnico o un grupo mafioso) y, una vez que comprende los procesos que están teniendo lugar, construye -con los sectores interesados de la comunidad-soluciones permanentes que involucran a las agencias del gobierno, a la propia comunidad y a los medios. (Montbrun, 2002, p. 8)

A diferencia de los modelos tradicionales, las nuevas formas de gestión del cuerpo policial implican un principio de proactividad, con el fin de que el policía cuente con la capacidad necesaria para comprender las implicaciones de la ejecución del PNVCC dentro de cada cuadrante. Es a partir del compromiso y el proceso de capacitación previo (antes de entrar en contacto directo con las comunidades) que pueden llegar a operar asertivamente, por medio de acciones preventivas en aquellos contextos que ofrecen mayores posibilidades de acciones delictivas y criminales.

Para concluir, el PNVCC tuvo efecto en un primer momento de su implementación (2010-2013) sobre la reducción de acciones delictivas en las principales ciudades del país (FIP, 2012b, p. 25): generó una cultura de la relación policía-ciudadano, optimizó el actuar de la institución con las medidas internas (como la capacitación del ONVCC a sus miembros) y también con estrate- 
gias de acciones preventivas. Este plan se convirtió en una gran herramienta de seguridad ciudadana tanto en el país como en otros países latinoamericanos que emularon esta estrategia; sin embargo, las nuevas administraciones prefirieron tomar otras medidas y reconceptualizar el Plan. De tomas maneras, no hay que olvidar que este Plan sirvió para mejorar la relación ciudadano-policía y revitalizó el sentir de la Policía Nacional de Colombia.

\section{Referencias}

Búvinic, M., Morrison, A., \& Shifter, M. (1999). La violencia en América Latina y el Caribe: un marco de referencia para la acción. Banco Interamericano de Desarrollo.

Delmas-Marty, M. (1986). Modelos actuales de politica criminal. Centro de PublicacionesMinisterio de Justicia.

Frühling, H. (2003). Policía comunitaria y reforma policial en América Latina: ¿cuál es el impacto? Instituto de Asuntos Públicos; Universidad de Chile.

Fundación Ideas para la Paz. (2011a). Resultados preliminares de la evaluación de impacto del PNVCC.

Fundación Ideas para la Paz. (2011b). La nueva vigilancia por cuadrantes mejora la seguridad en las ciudades. ¿Oportunidad para los nuevos alcaldes? Foro Semana $n .^{\circ} 60$ [Siguiendo el conflicto: hechos y análisis]. http://archive.ideaspaz.org/images/Siguiendo60esp.pdf

Fundación Ideas para la Paz. (2012a). Seguimiento, evaluación y acompañamiento a la implementación del Plan Nacional de Vigilancia Comunitaria por Cuadrantes [Informe final]. Cámara de Comercio de Bogotá.

Fundación Ideas para la Paz. (2012b). Informe de evaluación de impacto del plan nacional de vigilancia comunitaria por cuadrantes. Metropolitanas de Bogotá, Medellín, Cali, Barranquilla, Cúcuta, Bucaramanga, Pereira y Cartagena. Agosto, Colombia.

Krug, E., Dahlberg, L., Mercy, J., Zwi, A., \& Lozano, R. (2003). Informe mundial sobre la violencia y la salud. Organización Panamericana de la Salud. http://www.paho.org/ Spanish/AM/PUB/Violencia_2003.htm

Llorente, M. V., Bulla, P., \& Castillo, J. (2011a). Seguimiento y evaluación de impacto del plan nacional de vigilancia comunitaria por cuadrantes de la policía nacional de Colombia. En J. Araya (Ed.), Experiencias en América Latina: el desafío de evaluar programas de seguridad ciudadana (pp. 25-32). Instituto de Asuntos Públicos; Centro de Estudios en Seguridad Ciudadana; Universidad de Chile.

Llorente, M., \& Rivas, A. (2004). La caída del crimen en Bogotá: una década de políticas de seguridad ciudadana. Banco Internacional de Reconstrucción y Fomento; Banco Mundial.

Montbrun, A. (2002). Policía y seguridad pública: modelos en marcha. La Ley - Gran Cuyo, $6(1), 1-12$. 
Policía Nacional. (2010a). Estrategia institucional para la seguridad ciudadana: Plan Nacional de Vigilancia Comunitaria por Cuadrantes (PNVCC).

Policía Nacional. (2010b). Modificación del PNVCC. Policía Nacional.

Policía Nacional. (2010c). Estrategia de la Policía Nacional para la consolidación de la seguridad ciudadana (T. 3). Policía Nacional.

Policía Nacional. (2014). Sistema de Prevención, Convivencia y Seguridad Ciudadana. Policía Nacional.

Quintero Cordero, S. (2020). Seguridad ciudadana y participación de las comunidades en América Latina. Revista Cientifica General José María Córdova, 18(29), 5-24. http:// dx.doi.org/10.21830/19006586.561

Quintero Cordero, S., \& González, M. (2017). La evolución de la seguridad en el Sistema Internacional Contemporáneo: un balance a partir de los casos de Estados Unidos y la Federación de Rusia. En A. Castillo Castañeda y C. Niño González (Eds.), Nociones sobre seguridad y paz en las relaciones internacionales contemporáneas (pp. 447-472). Ediciones USTA.

Quintero Cordero, S., \& Molina Orjuela, D. (2013). La ilegalidad: una herramienta de análisis para la naturaleza del conflicto en Colombia. Revista Via Inveniendi et Iudicandi, 8(1), 159-190. https://revistas.usantotomas.edu.co/index.php/viei/article/view/846

Ripoll de Castro, A., y Quintero Cordero, S. (2018). Regionalismo Latinoamericano: la Alianza del Pacífico y la Aladi: ¿̇integraciones complementarias o estancamiento de la integración latinoamericana? En E. Pastrana Buelvas \&, H. Blomeier (Eds.), La Alianza del Pacifico: ¿atrapada en el péndulo del regionalismo y del interregionalismo? (pp. 223-243). Ediciones Opciones Gráficas.

Risco, M. (2009) Policía comunitaria como propuesta alternativa a los mecanismos tradicionales de seguridad [Ponencia]. X Congreso Nacional de Sociología Jurídica, Córdoba.

Velázquez, F. (2000). La teoría del hecho punible en el derecho colombiano: perspectivas [Ponencia]. Simposio Internacional sobre Sentido y Contenidos del Sistema Penal en la Globalización, Bogotá. http://arapajoe.es/poenalis/dosmil.htm 